ARTICLES

\title{
Selective neural representation of objects relevant for navigation
}

\author{
Gabriele Janzen $^{1,2} \&$ Miranda van Turennout ${ }^{2}$
}

As people find their way through their environment, objects at navigationally relevant locations can serve as crucial landmarks. The parahippocampal gyrus has previously been shown to be involved in object and scene recognition. In the present study, we investigated the neural representation of navigationally relevant locations. Healthy human adults viewed a route through a virtual museum with objects placed at intersections (decision points) or at simple turns (non-decision points). Event-related functional magnetic resonance imaging (fMRI) data were acquired during subsequent recognition of the objects in isolation. Neural activity in the parahippocampal gyrus reflected the navigational relevance of an object's location in the museum. Parahippocampal responses were selectively increased for objects that occurred at decision points, independent of attentional demands. This increase occurred for forgotten as well as remembered objects, showing implicit retrieval of navigational information. The automatic storage of relevant object location in the parahippocampal gyrus provides a part of the neural mechanism underlying successful navigation.

Studies on the neural basis of navigation have consistently shown the hippocampus to be crucially involved in the creation of an allocentric spatial representation of our environment ${ }^{1-12}$. The parahippocampal gyrus, a brain region highly interconnected with the hippocampus, has been implicated in the encoding of objects-in-place during navigation $^{5,6,13}$, as well as in the processing of spatial visual scenes ${ }^{14,15}$. Successful navigation is facilitated by the presence of objects, or landmarks, at different locations along a route ${ }^{16-18}$. Not all objects along a route, however, are equally relevant for navigation. Whereas objects at intersections convey information about which of the possible paths is the correct one, objects placed at simple turns in the road are of much less significance. Behavioral studies have reported that objects placed at decision points (that is, intersections) are more likely to be remembered later than objects placed at non-decision points ${ }^{17}$. They are also regarded as more important when participants evaluate the quality of a route description ${ }^{19}$. How this distinction between navigationally relevant and irrelevant objects is stored and maintained in the brain is still unknown. To date, all studies have focused on the neural correlates of encoding spatial information during navigation. To find one's way back in a surrounding, however, the information about relevant locations needs to be available at a later moment in time. Therefore, it is likely that spatial information that is crucial for pathfinding is encoded and stored differently than information that is of less importance. Here we report event-related fMRI evidence for differential representation of objects in the parahippocampal gyrus as a function of their navigational relevance in a large-scale environment.

In the study phase of the experiment, twenty healthy, right-handed human adults (10 female) watched a film sequence through a virtual museum, on one occasion outside the scanner. Participants were told that they were being trained to be a guide in the museum, and their task was to remember the objects and the route. The virtual museum consisted of two mazes of the same shape. In each maze, objects were placed on tables along the wall (Fig. 1a). Objects occurred either at an intersection (decision point objects) or at a simple turn (non-decision point objects, Fig. 1b). Half of the objects in the museum were toys, and the other half were objects from other semantic categories (non-toys). Participants were explicitly instructed to pay special attention to the toys to be able to guide a children's tour through the museum (see Methods). The placement of toys was counterbalanced between decision and non-decision points, resulting in a completely crossed factorial design with an equal number of attended objects (toys) and non-attended objects (non-toys) occurring at both decision and non-decision points (see Methods). This study design allowed us to distinguish between those effects specifically due to navigational relevance of object location (decision versus non-decision point objects) and effects related to simply paying more attention to one group of objects as compared to the other (toys versus non-toys).

After the study phase, fMRI time series were obtained while participants performed a simple object recognition task. Participants were presented with objects that had been shown in the museum, randomly intermixed with novel toys and novel non-toys. They indicated by button press whether or not they had seen the object in the film sequence. Importantly, in the recognition task, all objects were shown from a canonical perspective on a white background (Fig. 1c). Thus, during scanning, no maze-related information was presented. Differences in brain activity for decision and non-decision point objects could, therefore, only be explained by differences in navigational relevance during the study phase. Scrambled objects were included to serve as a low-level visual baseline (see Methods). All stimuli were presented rapidly, in a randomly intermixed order,

${ }^{1}$ Max Planck Institute for Psycholinguistics, Postbus 310, 6500 AH Nijmegen, The Netherlands. ${ }^{2}$ F.C. Donders Centre for Cognitive Neuroimaging, Box 9101,6500 HB Nijmegen, The Netherlands. Correspondence should be addressed to G.J. (gabriele.janzen@mpi.nl).

Published online 16 May 2004; doi:10.1038/nn1257 
to prevent participants from anticipating and changing strategies for the different types of objects. Our results show that neural activity in the parahippocampal gyrus reflects the navigational relevance of an object's previous location.

\section{RESULTS}

\section{Behavioral results}

Task performance was above chance level. No significant differences in error rates (misses) were observed for objects placed at decision (32.98\%) and non-decision (30.97\%) points, or for toys $(29.74 \%)$ and non-toys $(32.62 \%)$. False-positive responses were $15.71 \%$ for the novel toys and $18.09 \%$ for the novel nontoys. Scrambled objects evoked $1.82 \%$ false positives.

Response times showed an effect of the attentional manipulation during study. An analysis of variance (ANOVA) of the recognition times showed a main effect of attention $\left(F_{1,19}=9.63, P<0.001\right)$. Recognition times were significantly faster for toys than for non-toys (mean response latencies were $944 \mathrm{~ms}$ and $964 \mathrm{~ms}$, respectively; $t_{19}=3.103$, $P<0.01)$. In addition, an interaction between attention and decision point was obtained $\left(F_{1,19}=7.71, P<0.01\right)$. Recognition times were significantly faster for toys placed at decision points as compared to non-decision points (mean response latencies were $935 \mathrm{~ms}$ and $954 \mathrm{~ms}$, respectively; $t_{19}=2.334$, $P<0.05)$. No significant difference was observed for non-toys placed at decision and at non-decision points (mean response latencies were $973 \mathrm{~ms}$ and $955 \mathrm{~ms}$ ).
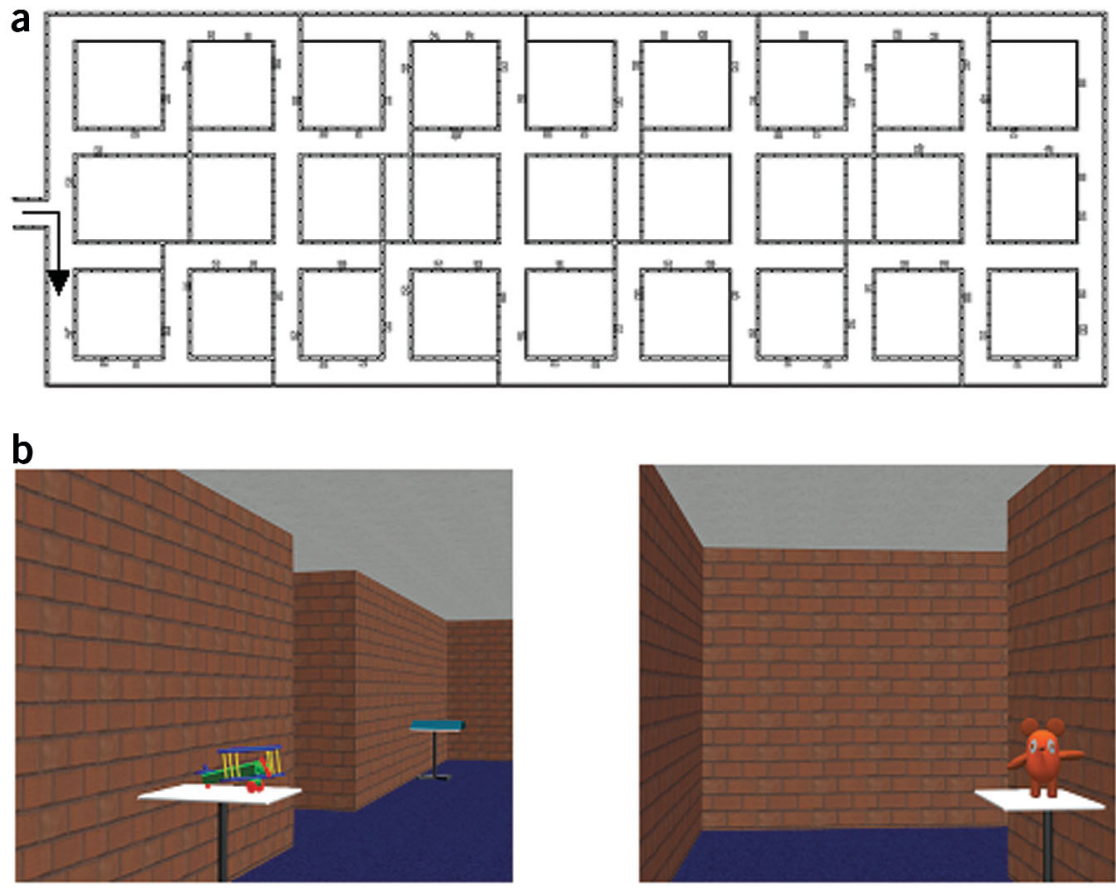

Toy at decision point

Toy at non-decision point

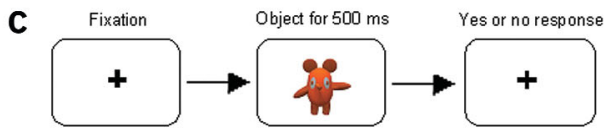

Figure 1 Virtual museum and recognition task. (a) The aerial perspective of the virtual museum. Participants started the tour in the direction of the arrow. The squares indicate tables on which the objects were placed. (b) Examples of scenes that participants viewed during the study phase. Shown here are two attended objects (toys) placed at a decision and a non-decision point from the viewpoint of the participants. (c) During a recognition task, participants were presented with objects from the virtual museum that were randomly intermixed with new objects, and then they indicated by button press whether or not they had seen the object in the prior film sequence.

\section{Functional brain imaging data}

Compared to a low-level visual baseline, objects strongly activated bilateral occipitotemporal cortices. This region is usually referred to as the ventral visual pathway and is known to be involved in object recognition ${ }^{20}$. In addition, we found increased activity in bilateral parahippocampal gyrus, in the left inferior frontal gyrus, and the left superior parietal lobe $(P<0.00001)$.

To test for effects of attention, we compared event-related fMRI responses to toys with those to non-toys. This comparison showed a significant increase in activity for toys in the right fusiform gyrus (Fig. 2a). There were no other brain regions showing increased activity for toys as compared to non-toys. An ANOVA of the averaged beta weights obtained for all voxels in this region (see Methods) showed a main effect of attention only (toys vs. non-toys; $F_{1,19}=19.48, P<$ 0.001 ). There was no main effect of decision point, and no interaction was obtained (Fig. 2b).

To examine the neural correlates of the navigational relevance of object location, we compared responses obtained for decision-point objects with responses obtained for non-decision point objects. This contrast revealed a focal increase in activity in the left and right parahippocampal gyri for decision-point objects (Fig. 3a). No such increase was observed in any other brain region. To dissociate effects of navigational relevance and effects that specifically resulted from paying attention to a certain object category, parahippocampal responses to toys were compared with parahippocampal responses to non-toys. In contrast to the effect of decision point, this comparison showed decreased neural activity for attended objects (toys) in the parahippocampal gyrus (Fig. 3b). Importantly, there was no significant interaction between the factors attention and object location.

To test whether the observed effect of navigational relevance reflected automatic changes in object processing ${ }^{21}$ or whether it was related to explicit memory processes ${ }^{22}$, we analyzed event-related responses in the parahippocampal gyrus separately for remembered and forgotten objects. Forgotten objects included all objects that participants had seen in the museum, but had indicated as 'not seen before' in the recognition test. Participants remembered $67 \%$ of the decision point objects and $69 \%$ of the non-decision point objects. Comparisons of the regional responses in the parahippocampal gyrus for decision and non-decision point objects showed a decision point-related increase in activity for remembered as well as forgotten objects (Fig. 4). For forgotten objects, however, the effect was significant in the right parahippocampal gyrus only.

Finally, we examined whether male and female participants showed differential effects of navigational relevance. To test for such differences, the beta weights obtained for the region of interest in the 
a
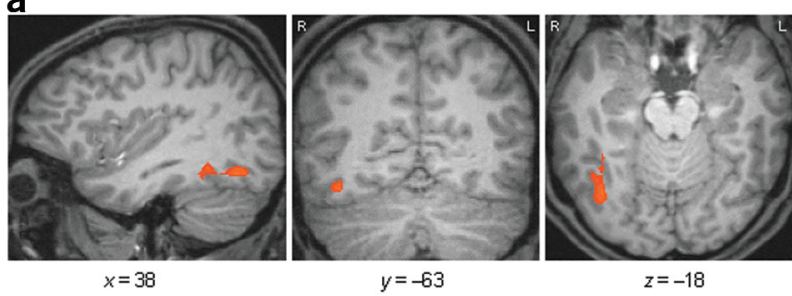

b

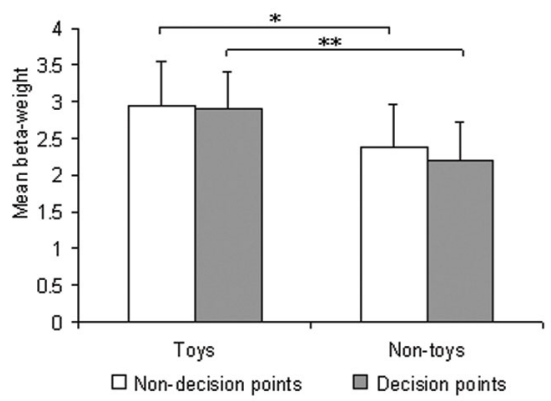

parahippocampal gyrus were entered in a repeated-measures ANOVA including the between factor sex (male and female) and the within factors object location (decision and non-decision point), object category (toys and non-toys) and hemisphere (right and left hemisphere). No significant effect of participant gender resulted.

\section{DISCUSSION}

Our data indicate that during route learning, the brain automatically distinguishes between objects at navigationally relevant and irrelevant locations. Representation of objects in the parahippocampal gyrus was directly related to their navigational relevance in a largescale environment. This selective neural marking for relevant objects occurred in the absence of any spatial information during retrieval, and even without conscious recollection of the route or conscious awareness of having seen the object previously.

Previous findings have emphasized the significance of the parahippocampal gyrus for object-place associations $s^{5,6,13,23}$. The parahip-

Figure 3 Decision-point versus non-decision-point objects. (a) Regions in the left and right parahippocampal gyrus showing increased activity for decisionas compared to non-decision-point objects, averaged across participants. Locations of peak activations, expressed in millimeters as Talairach coordinates ${ }^{36}$ were in the right $(x=+26, y=-39, z=-12 ; P<0.001)$ and in the left parahippocampal gyrus $(x=-25, y=-39, z=-11 ; P<0.001)$. The size of the region was larger in the right $\left(1,701 \mathrm{~mm}^{3}\right)$ than in the left hemisphere $\left(243 \mathrm{~mm}^{3}\right)$. No other brain regions showed enhanced responses. (b) Regional responses in the left and right parahippocampal gyrus showing increased activity for decision as compared to non-decision point objects. An ANOVA of the regionally averaged beta weights showed significant main effects of attention $\left(F_{1,19}=6.69, P<0.05\right)$ and decision point $\left(F_{1,19}=10.031, P<0.01\right)$. Whereas responses were greater for decision than for non-decision point objects in both the right $\left(t_{19}=2.975, P<0.01\right)$ and left parahippocampal gyrus $\left(t_{19}=2.534, P<0.05\right)$, the effect of attention was reversed: reduced responses were observed for toys as compared to nontoys (right hemisphere: $t_{19}=-2.12, P<0.05$; left hemisphere: $t_{19}=-2.58$, $P<0.05)$. There was no interaction between the factors attention and decision point $\left(F_{1,19}=0.058, P=0.812\right)$. Error bars reflect standard errors across participants.
Figure 2 Toys compared to non-toys. (a) A region in the right fusiform gyrus (BA 37) showing increased activity for attended objects (toys) compared to non-attended (non-toys). Location of peak activations, expressed in millimeters as coordinates in the Talairach and Tournoux brain atlas ${ }^{36}$ was $x=+38, y=-63, z=-18, P<0.00002$. (b) Regionally averaged beta weights for the right fusiform gyrus. $T$-contrasts showed a significant effect for toys compared to non-toys for both decision-point objects $\left(t_{19}=3.96\right.$, $P<0.001)$ and non-decision-point objects $\left(t_{19}=2.56, P<0.05\right)$. Error bars reflect standard errors across participants.

pocampal gyrus is relevant for the encoding of object locations, but not for encoding location alone ${ }^{23}$. Results also show that this region is not involved in object processing alone, so the encoding of objects-inplace is seen as a critical factor ${ }^{5}$. Importantly, however, our data clearly show that the parahippocampal activity is modulated by the specific navigational relevance of an object's location, even with showing objects in isolation. Objects previously placed at locations important for navigation generated more neural activity than did objects previously placed at locations with no navigational relevance. This neural marking of an object that occurred at a relevant location demonstrates the importance of the parahippocampal gyrus for human object-location memory. Selective representation of decisionpoint objects could be a part of the neural mechanism underlying successful navigation. Further studies involving interactive navigation in rich visual environments should provide additional evidence for such a mechanism.

Importantly, we showed that the decision point-related activity in the parahippocampal gyrus was independent of participants' attention to an object. An alternate explanation of the decision-point effect could have been that during the study phase, participants paid more attention to objects at decision points, and therefore, these objects induced more activity during later recognition. To rule out this alternate explanation, we included a control condition in which attention was explicitly modulated. Behavioral results showed that this manipulation was effective: shorter reaction times were observed for toys as compared to non-toys. The brain imaging data showed increased activity in the right fusiform gyrus for the attended objects (toys) as compared to the non attended objects (non-toys). This finding is consistent with earlier reports associating the cognitive effort of paying attention with increased neural activity in ventral occipitotemporal
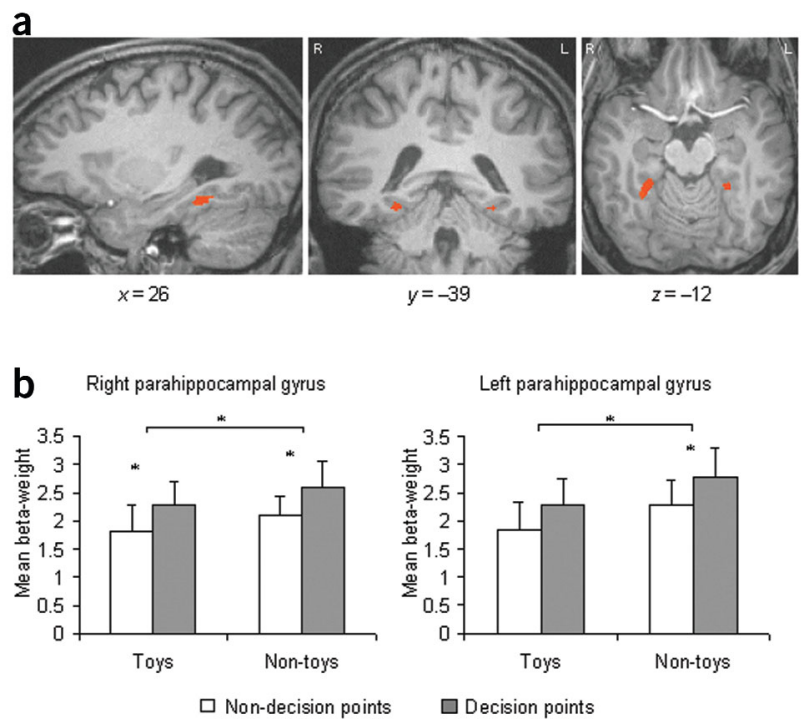
Figure 4 Remembered and forgotten objects. Parahippocampal responses were obtained for remembered and forgotten objects at decision and nondecision points. In the right parahippocampal gyrus, analysis of beta weights showed significantly larger responses for decision as compared to non-decision point objects, for both remembered and forgotten objects (remembered: $t_{19}=1.79, P<0.05$, forgotten: $t_{19}=2.37, P<0.01$ ). In the left parahippocampal gyrus, a significant decision point effect was observed for remembered objects $\left(t_{19}=2.57, P<0.01\right)$.

regions ${ }^{24-27}$. In the present study, however, attention was manipulated during the study phase, and not during the recognition task. The increase in fusiform activity could therefore indicate an effect of attention on later recognition. Importantly, activity in the right fusiform gyrus was only modulated by attention, and not by navigational relevance, indicating a dissociation between the two effects.

The pattern of results observed in the parahippocampal gyrus provided further evidence for differential effects of attention and navigational relevance. Whereas increased parahippocampal responses were observed for decision as compared to non-decision point objects, attended objects were associated with a decrease in activity in this region. This decrease could be related to better stimulus encoding during study for toys as compared to non-toys ${ }^{28,29}$. Alternatively, the decrease could reflect previously reported repetition-related changes in object representations ${ }^{30,31}$. The present data do not allow us to distinguish between these different explanations. Regardless of its underlying neural mechanism, however, the attention-related decrease in parahippocampal activity provides clear evidence for a dissociation between the effects of attention and decision point. The opposite direction of the attention and the decision-point effect eliminates the possibility that the decision point-related increase in parahippocampal activity simply results from paying more attention during learning.

Further strong support for this claim is provided by the absence of an interaction between the factors attention and navigational relevance: an identical increase in activity was observed for decisionpoint versus non-decision-point objects, regardless of whether they were attended or not (Fig. 3b). This dissociation between navigationally relevant object location and global attentional processes during route learning shows that the effect of decision points is highly automatic.

Furthermore, we tested whether the observed effect of navigational relevance reflected automatic changes in object processing, or whether it was related to explicit memory processes. We observed a decision point-related increase in activity for remembered as well as forgotten objects (Fig. 4). Thus, neural activity in the right parahippocampal gyrus reflected the navigational relevance of an object's location in the museum, even when participants did not remember having seen the object before. This independence of explicit memory strongly suggests that during pathfinding, the navigational relevance of an object is stored in the parahippocampal gyrus and is automatically activated once the object is encountered again.

Analyses of male and female participants showed no main effect. Previous results have shown sex differences, especially for spatial intelligence tasks ${ }^{32}$. However, meta- analyses showed that these differences are highly overrated ${ }^{33}$. According to other studies, male and female participants use different strategies while navigating ${ }^{34,35}$. A more recent fMRI study examining sex differences during navigation shows a differential neural network for men and women during maze exploration ${ }^{11}$. We did not find evidence for such differences between men and women during simple object recognition.

Our results show that the involvement of the parahippocampal gyrus in object-place associations is modulated by the navigational

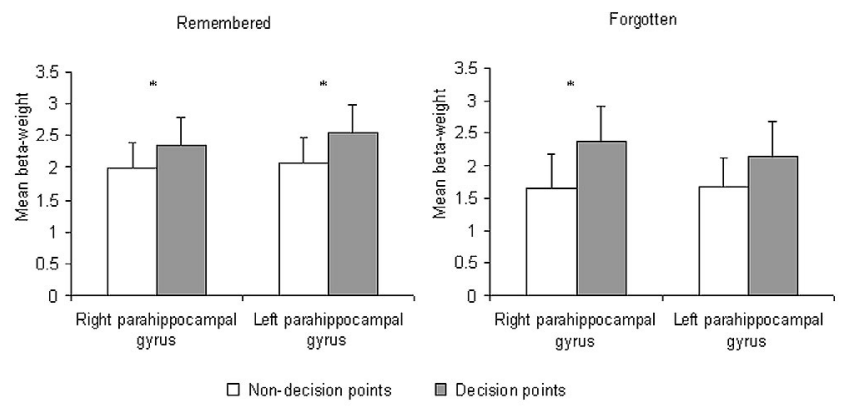

relevance of an object's location. We have the ability to find our way around in many different well-known, and also less well-known environments. Successful navigation through these environments is partly dependent on the automatic retrieval of spatial information connected to relevant object locations (e.g., turn left at the gas station). Our results show that this navigational information is acquired during route learning and stored in the parahippocampal gyrus for later use. This is a very rapid type of learning. Increased responses in the parahippocampal gyrus for navigationally relevant objects were observed after only one exposure to the maze. During later object recognition, this information is automatically activated, in the absence of spatial cues, and without explicit memory of having seen the object before. These rapidly induced changes in object representations in the parahippocampal gyrus allow for fast and dynamic updates of our spatial maps, necessary for pathfinding and navigation.

\section{METHODS}

Participants. Twenty healthy human adults (10 female) gave informed written consent before participating in the experiment. All participants were righthanded. Mean age was 23 years (range 18-30 years). The study was approved by the CMO Committee on Research Involving Human Subjects (Region Arnhem-Nijmegen).

Materials and procedure. The experiment was split in two parts: a study phase outside the scanner and a recognition phase during which functional images were acquired. Participants were given the following standardized written instruction for the study phase: "You apply for a job in a museum that exhibits belongings of famous people. You will be guided through two sections of the museum. The exhibits are placed on tables along the wall. Importantly, after training, you should be able to guide a children's tour through the museum. Therefore, while you are watching the film sequences pay special attention to toys and other things interesting for children."

The commercially available architecture software (3D TraumhausDesigner 4.0, Data Becker GmbH \& CO.KG) was used to create the film sequence through a virtual reality museum presented on a $1.60 \mathrm{GHz}-\mathrm{M}$ Pentium 4 personal computer with $512 \mathrm{MB}$ of RAM and a 15.0" XGA LCD screen. The virtual museum consisted of two mazes of the same shape. In each maze, there were 72 objects placed on tables along the wall (Fig. 1a). In total, 144 different objects were included in the mazes. Two mazes were used to reduce route complexity. In real-world dimensions, each maze had a length of 279 feet and was 112 feet wide in relation to a simulated eye level of 5.6 feet. The two mazes were shown in separate film sequences, lasting $8.5 \mathrm{~min}$ each. The order in which the two film sequence were presented was counterbalanced across subjects.

Objects occurred at decision points or at non-decision points (Fig. 1b). Thus, in the film sequence, a turn was made at both decision and non-decision points. This way, the effects of motion were not confounded with those of navigational relevance. Participants had no control over the timing in the virtual environment to ensure that the amount of time spent at decision and nondecision points was matched (an object was visible on average $5 \mathrm{~s}$ in the vocal focus and $11 \mathrm{~s}$ total). 
Twenty-five minutes after the study phase, fMRI time series were obtained while participants performed a simple object-recognition task. In this recognition phase, participants were instructed to decide as accurately and as quickly as possible whether they had seen the object in the former film sequences by pressing either a yes or a no response key. They reacted with their right hand, and responses were given by the index and the middle finger. A trial consisted of a fixation cross centered on the screen, followed by an object for $500 \mathrm{~ms}$ shown from a canonical perspective on a white background (Fig. 1c). Thus, during scanning, no maze-related information was presented. The average inter-stimulus interval was $4,000 \mathrm{~ms}$, jittered between $3,000 \mathrm{~ms}$ and 5,000 ms in steps of $250 \mathrm{~ms}$, counterbalanced over conditions. A total number of 252 stimuli were included in the recognition task. All stimuli were presented rapidly, in a randomly intermixed order to prevent participants from anticipating and changing strategies for the different event types. The entire stimulus material consisted of seven sets of 36 objects each, belonging to the following seven event types: decision objects toys, non-decision objects toys, decision objects non-toys, non-decision non-toys, novel toys, novel non-toys and scrambled objects. The scrambled objects were constructed from experimental objects using mosaic scrambling. All sets of objects were matched for word frequency.

fMRI data analyses. Functional images of the whole brain (455) were acquired on a 3-tesla MRI system (Siemens TRIO). Using a gradient-echo echo planar scanning sequence, 36 axial slices were obtained for each subject (voxel size $3 \times$ $3 \times 3 \mathrm{~mm}^{3}, \mathrm{TR}=2,268 \mathrm{~ms}$, field of view $=192, \mathrm{TE}=30 \mathrm{~ms}$, flip angle $=75^{\circ}$ ). All functional images were acquired in one run that lasted for $17 \mathrm{~min}$. After the acquisition of functional images, a high-resolution anatomical scan (T1weighted MP-RAGE, 176 slices) was acquired.

fMRI data were analyzed with BrainVoyager 2000 (Brain Innovation). Functional images were corrected for motion and slice scan time acquisition. Data were temporally smoothed with a high-pass filter removing frequencies below 3 cycles per time course. Functional images were coregistered with the anatomical scan and transformed into Talairach coordinate space using the nine-parameter landmark method of Talairach and Tournoux ${ }^{36}$. Images were spatially smoothed with a full-width at half-maximum (FWHM) Gaussian kernel of $6 \mathrm{~mm}$.

Statistical analyses were performed in the context of the general linear model, including seven effects of interests and confounds. Event-related hemodynamic responses for each of the different event types were modeled as delta functions convolved with a synthetic hemodynamic response function. Both fixed and random-effects group analyses were performed. The statistical threshold was set at $P<0.001$ at the voxel level, uncorrected for multiple comparisons. Region-of-interest analyses of the parahippocampal region were performed as follows: first, we functionally defined the parahippocampal region by selecting all voxels showing an effect of decision versus non-decision points in the group analysis. The right fusiform region was defined by the comparison between toys and non-toys. Then we obtained the beta weights (i.e., the regression coefficients) as indexes of effect size for all voxels included in these regions of interest, separately for all individual subjects, for each event type. These regionally averaged beta weights were analyzed in repeated-measurement ANOVAs. Specific effects were tested by applying $t$-contrasts to the regionally averaged beta weights obtained for the different event types.

\section{ACKNOWLEDGMENTS}

We thank G. Fernández, P. Hagoort and M. Coles for helpful discussions and their comments on the manuscript.

\section{COMPETING INTERESTS STATEMENT}

The authors declare that they have no competing financial interests.

Received 26 January; accepted 29 April 2004

Published online at http://www.nature.com/natureneuroscience/

1. Maguire, E.A. et al. Knowing where and getting there: a human navigation network. Science 280, 921-924 (1998).

2. Burgess, N., Jeffery, K.J. \& O'Keefe, J. (eds.) The Hippocampal and Parietal Foundations of Spatial Cognition (Oxford Univ. Press, Oxford, 1999).
3. O'Keefe, J. \& Dostrovsky, J. The hippocampus as a spatial map. Preliminary evidence from unit activity in the freely moving rat. Brain Res. 34, 171-175 (1971).

4. O'Keefe, J. \& Nadel, L. The Hippocampus as a Cognitive Map (Oxford, Clarendon, 1978).

5. Maguire, E.A., Frith, C.D., Burgess, N., Donnett, J.G. \& O’Keefe, J. Knowing where things are: parahippocampal involvement in encoding object locations in virtual large-scale space. J. Cogn. Neurosci. 10, 61-76 (1998).

6. Aguirre, G.K., Detre, J.A., Alsop, D.C. \& D'Esposito, M. The parahippocampus subserves topographical learning in man. Cereb. Cortex 6, 823-829 (1996).

7. Ekstrom A.D. et al. Cellular networks underlying human spatial navigation. Nature 425, 184-187 (2003).

8. Maguire E.A. et al. Navigation-related structural change in the hippocampi of taxi drivers. Proc. Natl. Acad. Sci. USA, 97, 4398-4403 (2000).

9. Burgess, N., Maguire, E.A. \& O'Keefe, J. The human hippocampus and spatial and episodic memory. Neuron 35, 625-641 (2002).

10. Wood, E., Dudchenko, P.A., Robitsek, R.J. \& Eichenbaum, H. Hippocampal neurons encode information about different types of memory episodes occurring in the same location. Neuron 27, 623-633 (2000).

11. Grön, G., Wunderlich, A.P., Spitzer, M., Tomaczak, R. \& Riepe, M.W. Brain activation during human navigation: gender-different neural network as substrate of performance. Nat. Neurosci. 3, 404-408 (2000).

12. Dragoi, G., Harris, K.D. \& Buzsáki, G. Place representation within hippocampal networks is modified by long-term potentiation. Neuron 39, 843-853 (2003).

13. Duezel, E. et al. Human hippocampal and parahippocampal activity during visual associative recognition memory for spatial and nonspatial stimulus configurations. J. Neurosci. 23, 9439-9444 (2003).

14. Epstein, R. \& Kanwisher, N. Cortical representation of the local visual environment. Nature 392, 598-601 (1998).

15. Bar, M. \& Aminoff, E. Cortical analysis of visual context. Neuron 38, 347-358 (2003).

16. Golledge, R.G. (ed.) Wayfinding Behavior (Johns Hopkins Univ. Press, Baltimore, 1999).

17. Blades, M. \& Medlicott, L. Developmental differences in the ability to give route directions from a map. J. Environ. Psychol. 12, 175-185 (1992).

18. Johnsrude, I.S., Owen, A.M., Crane, J., Milner, B. \& Evans, A.C. A cognitive activation study of memory for spatial relationships. Neuropsychologia 37, 829-841 (1999).

19. Daniel, M.-P. \& Denis, M. Spatial descriptions as navigational aids: a cognitive analysis of route directions. Kognitionswissenschaft 7, 45-52 (1998).

20. Ungerleider, L.G. \& Mishkin, M. Two cortical visual systems. in Analysis of Visual Behavior (eds. Ingle, D.J., Goodale, M.A. \& Mansfield, R.J.W.) 549-586 (MIT Press, Cambridge, Massachusetts, 1982).

21. Hasher, L. \& Zacks, R.T. Automatic and effortful processes in memory. J. Exp. Psychol. Gen. 108, 356-388 (1979).

22. Caldwell J.L. \& Masson, M.E.J. Conscious and unconscious influences of memory for object location. Mem. Cognit. 29, 285-295 (2001).

23. Owen, A.M., Milner, B., Petrides, M. \& Evans, A.C. A specific role for the right parahippocampal gyrus in the retrieval of object-location: a positron emission tomography study. J. Cogn. Neurosci. 8, 588-602 (1996).

24. Saenz, M., Buracas, G.T. \& Boynton, G.M. Global effects of feature-based attention in human visual cortex. Nat. Neurosci. 5, 631-632 (2002).

25. Yantis S. et al. Transient neural activity in human parietal cortex during spatial attention shifts. Nat. Neurosci. 5, 995-1002 (2002).

26. Kanwisher, N. \& Wojciulik, E. Visual attention: insights from brain imaging. Nat. Rev. Neurosci. 1, 91-100 (2000).

27. Vuilleumier, P., Armony, J.L., Driver, J. \& Dolan, R.J. Effects of attention and emotion on face processing in the human brain: an event-related fMRI study. Neuron 30, 829-841 (2001).

28. Gabrieli, J.D.E., Brewer, J.B., Desmond, J.E. \& Glover, G.H. Separate neural bases of two fundamental memory processes in the human medial temporal lobe. Science 276, 264-266 (1997).

29. Brewer, J.B., Zhao, Z., Desmond, J.E., Glover, G.H. \& Gabrieli, J.D.E. Making memories: brain activity that predicts how well visual experience will be remembered. Science 281, 1185-1187 (1998).

30. Van Turennout, M., Ellmore, T. \& Martin, A. Long-lasting cortical plasticity in the object naming system. Nat. Neurosci. 3, 1329-1334 (2000).

31. Henson, R., Shallice, T. \& Dolan, R. Neuroimaging evidence for dissociable forms of repetition priming. Science 287, 1269-1272 (2000).

32. Maccoby, E.E. \& Jacklin, C.N. The Psychology of Sex Differences (Stanford University Press, Stanford, California, 1974).

33. Hyde, J.S. How large are cognitive gender differences? A meta-analysis using $w^{2}$ and d. Am. Psychologist 36, 892-901 (1981).

34. Layton, C.A. Gender differences in wayfinding-strategies: relationship to spatial ability and spatial anxiety. Sex Roles 30, 765-779 (1994).

35. Sandstrom, N.J., Kaufman, J. \& Huettel, S.A. Males and females use different distal cues in a virtual environment navigation task. Cogn. Brain Res. 6, 351-360 (1998).

36. Talairach, J. \& Tournoux, P. A Co-Planar Stereotaxic Atlas of the Human Brain (Thieme Medical Publishers, New York, 1988). 